\title{
MAC Protocols for Multirate OV-CDMA System in Optical Packet Network: a comparative Approach
}

\author{
Elie Inaty \\ Faculty of Eng. \\ University of Balam and, Lebanon \\ elie.inaty@bal amand.edu.1b
}

\author{
Robert Raad \\ Faculty of Eng. \\ Laval University, Canada \\ robert.raad.1@ulaval.ca
}

\author{
Paul Fortier \\ Faculty of Eng \\ Laval University, Canada \\ fortier@gel.ulaval.ca
}

\author{
Hossam M. H. Shalaby \\ Faculty of Eng. \\ University of Alexanderia, Egypt \\ shalaby@ieee.org
}

\begin{abstract}
In this paper, we compare the performance of two newly proposed MAC protocols for multirate optical CDMA (OCDMA) networks. The first protocol is the S-ALOHA/OFFHCDMA and the second is the $R^{3}$ TOFFH-CDMA. The main subject in this proposal is to exploit the potential of the optical fast frequency hopping CDMA using fiber-Bragg grating when jointly used with two different MAC protocols in a link layer as an effective way of integrating multirate traffic. The system throughput and the average packet delay are compared for both systems. It is shown that the S-ALOHA is better than the $R^{3} T$ when the user's activity and the offered load are high while the $R^{3} T$ is better for smaller values. Both protocols can be competitive in terms of the system throughput with the advantage is for the $R^{3} T$ protocol at moder ate offered load. However, the $R^{3} T$ protocol suffers a higher delay mainly because of the presence of additional modes.
\end{abstract}

Keywords-S-ALOHA, overlapped CDMA, fiber-Bragg grating, optical link layer, $M A C$ protocol, $R^{3} T$.

\section{INTRODUCTION}

Asynchronous optical code division multiple-access (OCDMA) has gained more interest recently [1]-[3] due to its excess bandwidth that offers to serve the ever-increasing network dimension, especially in the presence of diverse multimedia traffies in today's communication systems. This, in turn, requires more complex control mechanism to handle the flow of information in such network [4]. For this reason, we should impose certain rules on the transmission and the reception of information packets. This is usually performed at the link layer of the optical network [4]-[6]. We refer to such control mechanism as multiple-access control (MAC) protocols.

Two MAC protocols namely slotted ALOHA (S-ALOHA) [4][5][7] and round-robin receiver/transmitter $\left(R^{3} T\right)$ [8] have been lately proposed and analyzed for OCDMA systems in optical packet networks. Our objective is to compare the performance of two multirate optical fast frequency hopping code division multiple access (OFFH-CDMA) systems controlled at the link layer by the proposed protocols. These two multirate systems are the variable processing gain (VPG) OCDMA [7] and the overlapped OCDMA (OV-CDMA) [9] systems.

This paper is organized as follows. Section II introduces the system model for both protocols. The packet correct probability is derived in Section III. Section IV presents the performance evaluation of the two protocols in terms of the throughput and the average packet delay. Numerical results are covered in Section V. Finally, concluding remarks end the paper in Section VI.

\section{SYSTEM MODEL}

Consider the OFFH-CDMA communication network proposed in [9] where $K$ users are exploiting this network in a star topology. This system makes use of fiber Bragg gratings (FBG) that slice passively and temporally the incoming pulse to perform the encoding and the decoding operations for each terminal user. The round-trip time between two consecutive gratings determine the chip duration $T_{c}$ and number of grating $G$ with the chip duration determines the nominal bit duration $T_{n}=G T_{c}$. Consequently, the nominal transmission rate is defined as $R_{n}=l / T_{n}$. Each user is assigned a unique code. We assume in this architecture that each user is equipped by a fixed transmitter and a tunable receiver (FTTR). Consider that the time is partitioned into equal intervals or slots. Each station initiates its transmission at the beginning of the time slot. We assume that the slot duration equals to the length of the packet, i.e. $T_{p}=L G T_{c}$, where $L$ designates the number of bits per packet.

\section{A. S-ALOHA/OFFH-CDMA:}

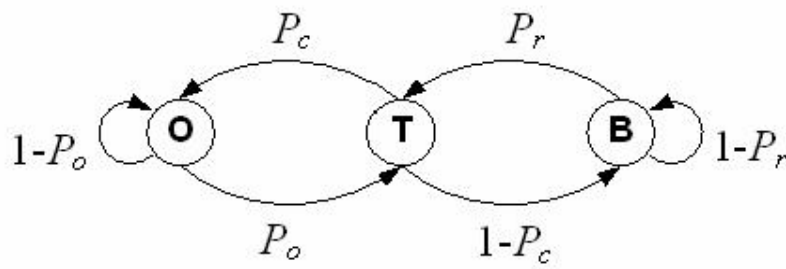

Fig. 1: State diagram of the S-ALOHA protocol

The S-ALOHA/OFFH-CDMA had been analyzed in detail in [7]. Initially, the system is empty and users generate $\xi_{0}$ new packets with probability $P_{0}$. Transmitted over the optical channel, some packets will be received correctly with a probability $P_{c}$ and some will be received in error due to collision and MAI. The terminal whose packets are incorrectly received is backlogged and it retransmits the packets after a random delay with a probability $P_{r}$. At the next time slot, the 
arrivals are composed of new packets and retransmitted packets. In general, the composite arrivals are modeled as a Markov chain of three states representing three modes: origination mode $(\mathrm{O})$, transmission mode $(\mathrm{T})$, and backlogged mode (B). Each user can be in one of the three modes at a time as described in Fig. 1.

\section{B. $R^{3}$ T/OFFH-CDMA:}

Beside the S-ALOHA protocol, we attempt to control the packet flow of users using the $R^{3} T$ protocol [8]. We assume that a packet corresponds to one bit and $L$ bits form a message so that the message length is equal to the packet length in SALOHA. Each user transmits his message with a probability $A$, called user's activity. This message is stored in a single buffer, freed once the message is sent correctly. Any message that arrives concurrently at a non-empty buffer will be discarded. The terminal behavior is described by the block diagram shown in Fig. 2. The user wishing to transmit his packets sends a request to the destination station at first, and therefore he is in the requesting mode. The receiver of the destination station scans across all codes in a round-robin fashion, switches to the desired code signature at the next time slot and sends acknowledgements to the transmitting station to proceed on its transmission. At this time, the receiving station enters the reception mode.

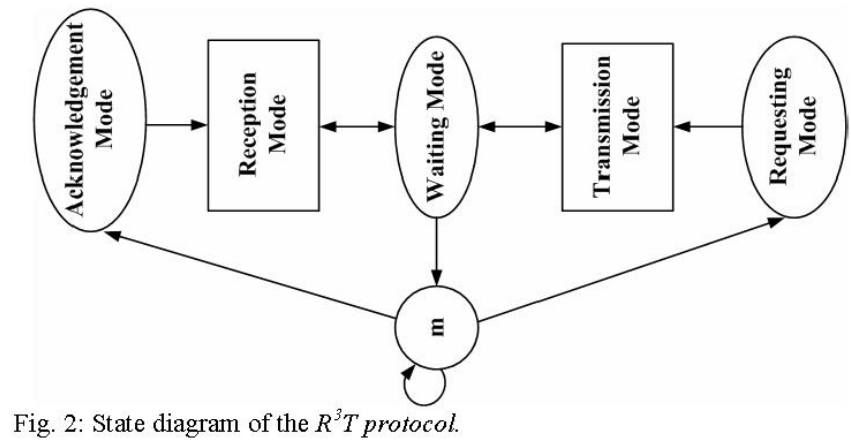

Before expiring the timeout duration $\tau_{o}$, if the transmitting station receives the acknowledgement, it enters the transmission mode and transmits the first $t$ packets following the go-back $n$ protocol [8], where $n=t$ is the two-way propagation time in slots between the transmitting and receiving stations. It can be $t \leq L$ or $t \geq L$. It then enters the waiting mode, waiting for acknowledgements. If no acknowledgements after the time out duration, the station retransmits its last $t$ packets in a round robin fashion as well. Once the message is received correctly and the station has nothing to transmit, it returns to its initial state $m$. At the reception side, once a packet is received correctly, an acknowledgement is being sent. Otherwise, the receiving station sends an ask-for-retransmission to the transmitting station and enters the waiting mode. If there is a message arrival and there is no request, then the receiving station asks the transmitting one for a connection request. If the latter does not respond, the receiving station returns to its initial state $m$.

\section{PACKET CORRECT PROB ABILITY}

In this paper we consider that all users employ ON-OFF keying (OOK) modulation scheme to transmit their data. In addition, the bit error rate (BER) is obtained from simulation using the Extended Hyperbolic Congruential Codes (EHC) [10] and it is given by $P_{b}$.

Assume a fixed packet time duration of $T_{p}=L T_{n}=L G T_{c}$ where $L$ is the nominal packet length. In [9], we have shown that due to the linearity of the encoder-decoder set, multi-bits will be coded and transmitted when the data rate increases beyond $R_{n}$ as shown in Fig. 3 ( $\lambda_{i}$ represents the $i^{\text {th }}$ wavelength in the desired user code). At the receiver end, the decoder observes practically multicode, which are delayed according to the transmission rate of the source. Accordingly, to increase the number of bits per packet of fixed length $L$, we increase the source transmission rate above the nominal rate without decreasing the PG as in the previous system. When a terminal transmits at rate $R_{s}>R_{n}$, it introduces a bit overlap coefficient $\varepsilon_{s}$, which represents the number of overlapping chips between two consecutive bits [7]. Accordingly the new bit rate is related to $R_{n}$ throughout the following equation

$$
R_{s}=\frac{G}{G-\varepsilon_{s}} R_{n}
$$

Let $\varepsilon_{s}$ be the overlapping coefficient. In addition, let $X_{b}^{(O)}$ to be the total number of overlapped bits in a packet time slot, which is given by [7]:

$$
X_{b}^{(0)}=\left\lfloor\frac{L G-\varepsilon_{s}}{G-\varepsilon_{s}}\right\rfloor
$$

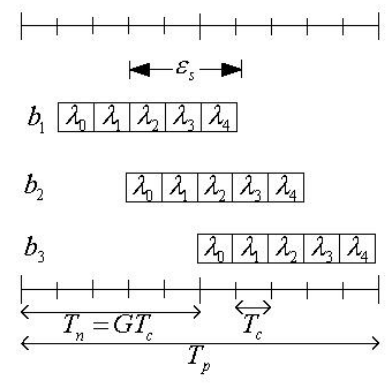

a)

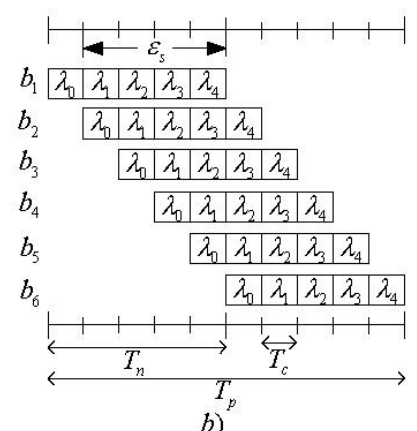

b)
Fig. 3: Optical Overlapped OFFH-CDMA packet model of a single user in a given time slot. a) $\varepsilon_{s}=3$, b) $\varepsilon_{o}=4$

Consequently, the rate in a packet network will be

$$
R_{s}=\frac{X_{b}^{(O)}}{L} R_{n}(\text { bits } / \mathrm{sec})
$$

Fig. 3 illustrates an example of the overlapping process in a packet time slot. In this example, the packet length is $L=2$, and the PG is $G=5$. When the overlapped coefficient is increased to $\varepsilon_{s}=3$ as shown in a), the transmission rate is increased to three bits per packet. On the 
other hand, b) shows the case where $\varepsilon_{s}=4$. Accordingly, the transmission rate is six bits per packet.

Since $P_{b}(i)$ differs at every bit position, then the packet correct probability is given by

$$
\left.P_{o}(K)=\prod_{i=0}^{X_{b}^{(0)}} 1 \text { [ } 1-P_{b}(i)\right]
$$

\section{PERFORMANCE EVALUATION}

The equilibrium point analysis (EPA) [8] is used to measure the performance of both protocols (intermediate derivations are omitted due to the limited space). At an equilibrium point of the system, the expected number of stations entering a state in any time slot is equal to that departing from it.

\section{A. $\quad S-A L O H A / O F F H-C D M A$ :}

$\mathrm{We}$ assume the following system parameters: the retransmission probability $P_{r}=B$, an origination probability $P_{0}=\alpha$, the number of packets in a message is geometrically distributed with mean $l$. in addition if a packet is not correctly received, it will be backlogged, then retransmitted in the next time slot with a random delay $d_{t}$ slots. On the other hand a receiver when tuned to a particular code, it receives a message with probability $\sigma$.

The steady state throughput (defined as the number of successfully received packets per time slot) is given by

$$
\beta(K, \alpha, \sigma, B, t)=\frac{B S_{0} P_{o}\left(S_{0}\right)}{1+B-P_{o}\left(S_{0}\right)}
$$

Where $S_{0}$ can be obtained such that the following equality is satisfied

$$
\frac{K}{S_{0}}=\frac{B(t-1) \sigma P_{c}\left(S_{0}\right)}{\alpha(1-\sigma)[1+B(t-2)]\left[1+B-P_{o}\left(S_{0}\right)\right]}+1
$$

The average offered traffic can be estimated by

$$
R=(K-\bar{n}) \alpha+\bar{n} B
$$

Where, $\bar{n}$ is the expected number of backlogged packets at equilibrium, and it is given by

$$
\bar{n}=\frac{S_{0}\left[1-P_{o}\left(S_{0}\right)\right]}{1+B-P_{o}\left(S_{0}\right)}
$$

The steady state delay according to Little's theorem can be written as

$$
D=\frac{\bar{n}}{\beta(K, \alpha, \sigma, B, t)}
$$

\section{B. $R^{3} T / O F F H-C D M A$ :}

The system throughput under the $R^{3} T$ is computed in [8] as

$$
\begin{aligned}
& \beta\left(K, A, t, \tau_{o}, L\right)= \\
& \frac{P_{o}(K) \cdot L \cdot r_{o}}{L+\left(1-P_{o}(K)\right)(\min \{t, L\}-1)(L-\min \{t, L / 2\})}
\end{aligned}
$$

where $r_{o}$ can be obtained such that the following condition is satisfied:

$$
\begin{aligned}
& K\left[L+\left(1-P_{o}(K)\right)(\min \{t, L\}-1)(L-\min \{t, L / 2\})\right]= \\
& r_{o}\left[\begin{array}{l}
2 t L\left(1-P_{c}\left(r_{o}\right)\right)+(2 t+2 L-1) P_{c}\left(r_{o}\right)+\frac{P_{c}\left(r_{o}\right)}{\rho} \\
\left.+A(t-1) \frac{1-\rho}{\rho} P_{o}\left(r_{o}\right)+\left\{1-\left[1-\frac{\rho}{A(1-\rho)}\right]^{\frac{1}{\tau_{o}}}\right\}^{1} P_{o}\left(r_{o}\right)\right]
\end{array}\right.
\end{aligned}
$$

where

$$
\rho=\rho\left(r_{o}\right)=0.5\left[\sqrt{u^{2}+4 u}-u\right]
$$

and

$$
u=\frac{A P_{o}\left(r_{o}\right) \cdot \tau_{o} \cdot r_{o}}{K\left[L+\left(1-P_{o}\left(r_{o}\right)\right)(\min \{t, L\}-1)(L-\min \{t, L / 2\})\right]} .
$$

The delay is given by

$$
D=\frac{K \cdot A}{\beta\left(K, A, t, \tau_{o}, L\right)}
$$

where $K A$ is the average offered traffic.

\section{RESULTS AND COMPARISON}

In this simulation we have assumed that the number of stations is $K=12$, the processing gain $G=31$, the packet length under S-ALOHA protocol is $L=300$ bits/time slot and the message is one packet. While equivalently, under the $R^{3} T$ protocol the packet length is one bit/time slot and $L=300$ designates the message length in packets. In the S-ALOHA, the retransmission probability $\mathrm{Pr}=0.9$; whereas in the $R^{3} T$, the packet transmission probability (user's activity) $\mathrm{A}=0.1$, $0.5,0.6$ and 1 , the time out duration $\tau_{0}=1$ time slot and the two-way propagation delay $t=8$ time slots in fiber lengths of $800 \mathrm{~m}$. In addition, we consider that both multirate systems transmit at a normalized rate of $R_{S}=714$ which corresponds to an increase of overlapping coefficient $\varepsilon_{s}$ from 0 to 18 for the overlapped system and a reduction of $\mathrm{PG}$ to 13 for VPG system. The throughput of both systems is present in Fig. 4. Notice that in both cases $R^{3} T$ protocol exhibits higher throughput than S-ALOHA protocol when the user's activity $A$ $<0.6$. As $A$ increases the throughput of $R^{3} T$ decreases compared to S-ALOHA. Note that for the $R^{3} T$ protocol the throughput curves reach around seven packets per slot for the overlapped system while they start declining after seven for VPG system given that the maximum offered load is twelve packets.

At moderate transmission rate, as $A$ increases the offered load also increases and the maximum reachable throughput is 
stabilizing around seven. This means that the $R^{3} T$ protocol always guarantees a successful transmission of its load. We remark in this case that both the VPG-OFFH-CDMA and the overlapped OFFH-CDMA systems have closer throughput under such protocol. For $A=1$ all the six users are transmitting at the same time, nevertheless the throughputs of both OCDMA systems are declining. This is because more users are now trying to transmit, while other users are still busy transmitting their long messages. So, the interference significantly increases and any unsuccessful transmission is replaced by the retransmission of the last $t$ packets and the optical channel is crowded by the retransmitted packets. This problem can be resolved in S-ALOHA protocol by randomly delaying the distorted packets, which are then retransmitted with a probability $P_{r}$. In this way contention can be efficiently controlled and the throughput is optimized. Notice also that under both protocols, the throughput of the overlapped system outperforms that of VPG system especially at higher propagation delay.

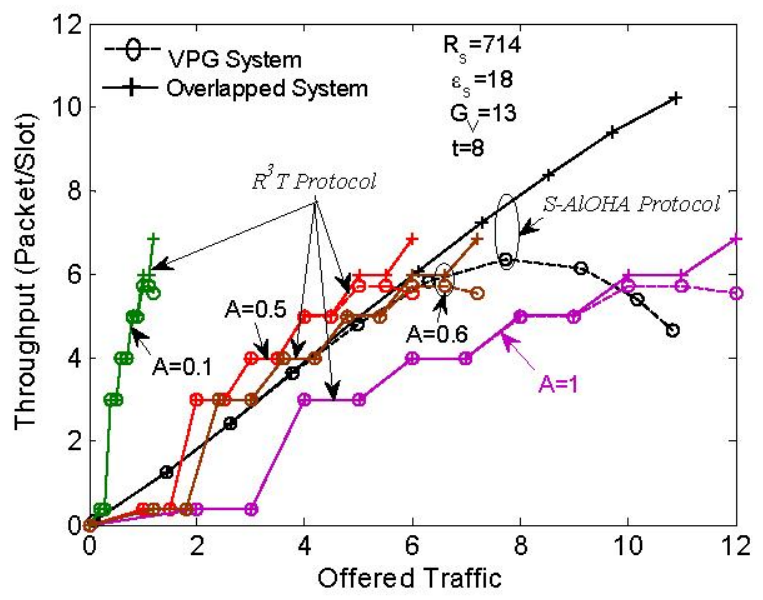

Fig. 4: The throughput versus the offered traffic for a two-way propagation time of eight time slots.

In Fig. 5 we present the average packet delay versus the system throughput of both multirate OCDMA systems operating under the two mentioned protocols. We remark that the $R^{3} T$ protocol exhibits higher delay, especially at low throughput even if the user's activity is reduced as revealed by the figure. This significant delay is caused by two factors: the adoption of go-back- $n$ protocol for the retransmission of the distorted packets where $n=t$ and the signaling packets such as request, acknowledgement and ask-for-retransmission, which in case of failure, place the system in the waiting mode for a period of time. Therefore, by minimizing the transmission activity of users $(A=0.1)$, the average system delay encountered by the $R^{3} T$ is still higher than that encountered by the S-ALOHA.

\section{CONCLUSION}

A comparison of performances of two multirate OCDMA systems under two different MAC protocols: the S-ALOHA and the $R^{3} T$ has been performed. It is shown that the $\mathrm{S}$ -
ALOHA is better than the $R^{3} T$ when the user's activity $A$ and the offered traffic are high while the $R^{3} T$ is better for smaller values of $A$ and moderate traffic. In addition, both protocols can be competitive in terms of system throughput for moderate offered traffic by fitting the corresponding parameters to an appropriate setting. However, the $R^{3} T$ protocol suffers a higher delay mainly because the presence of additional modes. Such modes are not available in S-ALOHA protocol. Finally, the overlapped OCDMA system always outperforms the VPG OCDMA system regardless the protocol used.

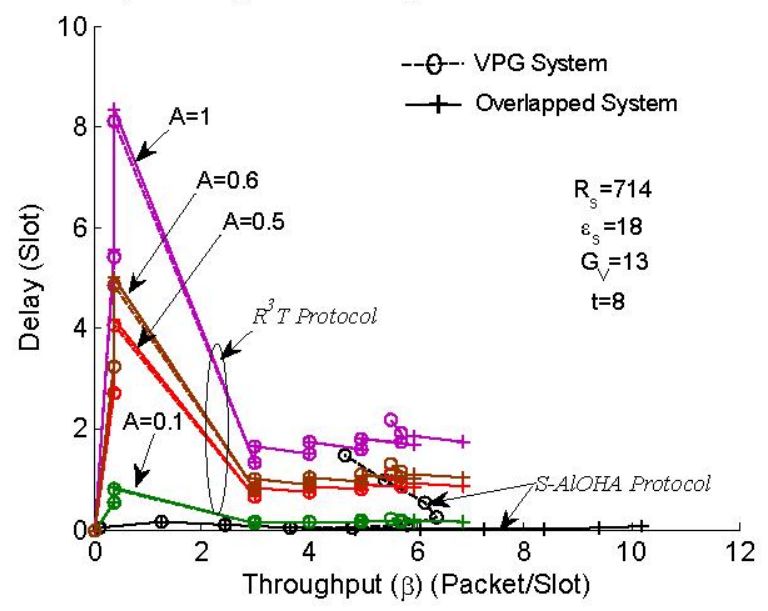

Fig. 5: The delay versus the throughput for a two-way propagation time of eight time slots.

\section{REFERENCES}

[1] S. Zahedi, and J. A. Salehi, "Analytical comparison of various fiberoptic CDMA receiver structures," IEEE J. Lightwave Technol., vol. 18, pp. 1718-1727, December 2000 .

[2] A. Stok and E. H. Sargent, "Comparison of diverse optical CDMA codes using a normalized throughput metric," IEEE Comm. Letters, vol. 7, pp. 242-244, May 2003.

[3] H. Ben Jaafar, S. LaRochelle, P. -Y. Cortes, and H. Fathallah, "1.25 Gbit/s transmission of optical FFH-OCDMA signals over $80 \mathrm{~km}$ with 16 users," OFC, vol. 2, pp TuV3.1 -, TuV3.3, 2001.

[4] P. Kamath, J. D. Touch and A. J. Bannister, "The need for media access control in optical CDMA networks," IEEE INFOCOM, vol. 4, pp 2208 - 2219, March 2004.

[5] D. Raychauhuri, "Performance analysis of random access packetswitched code division multiple access systems," IEEE. Trans. Comm., vol. COM-29, No. 6, pp 895-901, June 1981.

[6] C.-S. Hsu and V. O. K. Li, "Performance analysis of slotted fiber-optic code-division multiple-access (CDMA) packet networks," IEEE Trans. Commun., vol. 45, pp. 819-828, July 1997.

[7] R. J. Raad, E. Inaty, P. Fortier and H. M. H Shalaby, "Optical SALOHA/CMDA system for multirate application: system architecture and performance evaluation," IEEE Globecom., vol. 4, pp 1936 - 1941, December 2005.

[8] H. M. H. Shalaby, "Performance analysis of an optical CDMA random access protocol," J Lightwave Technol, vol. 22, pp. 1233-1241, May 2004.

[9] E. Inaty, H. M. H. Shalaby, and P. Fortier, "On the cutoff rate of a multiclass OFFH-CDMA system," IEEE Trans. Commun., vol.53, pp. 323-334, February 2005

[10] L. D. Wronski, R. Hossain, and A. Albicki, "Extended Hyperbolic Congruencial Frequency Hop Code: Generation and Bounds for Crossand Auto-Ambiguity Function," IEEE Trans. on Communications, vol.44, no.3, pp. 301-305, Apr 1996. 